\title{
Magnetic study of interatomic interactions, synthesis, structural and mass spectroscopy investigations of lanthanum gallate doped with cobalt and magnesium
}

\author{
D.A. Korolev*, N.V. Chezhina, S.I. Lopatin \\ St. Petersburg State University, Universitetskii pr. 26, St. Petersburg, Russian Federation
}

\section{A R T I C L E I N F O}

Article history:

Received 13 August 2014

Received in revised form 27 October 2014

Accepted 12 November 2014

Available online 21 November 2014

\section{Keywords:}

Ceramics

Magnetic properties

Powder diffraction

Secondary ion mass spectroscopy (SMS)

\begin{abstract}
A B S T R A C T
For the first time by X-ray method two phases of the solid solutions $\mathrm{LaCo}_{x} \mathrm{Ga}_{1-1.2 x} \mathrm{Mg}_{0.2 x} \mathrm{O}_{3-\delta}$ and $\mathrm{LaCo}_{x-}$ $\mathrm{Ga}_{1-1.5 x} \mathrm{Mg}_{0.5 x} \mathrm{O}_{3-\delta}(x=0.01-0.10)$ with different structure were found - rhombohedral and orthorhombic phases. On the basis of the data on evaporation of the components a synthetic procedure was advanced allowing the losses of cobalt to be minimized. The study of magnetic characteristics of obtained solid solutions showed the formation of high nuclearity clusters containing cobalt atoms, and also magnesium and associated vacancies even in diluted solid solutions. Clusters are characterized by a competition between ferro- and antiferromagnetic exchange interactions, whereas the long order exchange is antiferromagnetic.
\end{abstract}

(c) 2014 Elsevier B.V. All rights reserved.

\section{Introduction}

Solid electrolytes, ionic, electron-ionic conductors based on oxide matrices are of great practical importance in the technologies of solid oxide fuel cells (SOFC). Doped lanthanum gallate is one of the well-accepted subjects used in SOFC owing to a high mobility of oxygen ions and low coefficients of thermal expansion [1].

A number of works is devoted to the study of the structure and conducting properties of doped lanthanum gallate [2-5], to the search for the compositions providing a maximal ionic conductivity. Introduction of strontium and/or magnesium into lanthanum gallate often gives rise to secondary phases of the $\mathrm{LaSrGa}_{3} \mathrm{O}_{7}, \mathrm{LaSrGaO}_{4}$ and $\mathrm{La}_{4} \mathrm{Ga}_{2} \mathrm{O}_{9}$ oxides, which decreases the conductivity and life spans of the material. At the same time the introduction of transition metal cations along with strontium and magnesium was noted to result in single phase samples, i.e. is in aid to the stabilization of the structure of doped lanthanum gallate. Moreover, the concentration and nature of the dopants drastically affect the conductivity. Nickel and cobalt containing systems are considered to be the best conductors and chromium and manganese containing systems the worst [2,6-18]. The reasons of the influence of the dopants, as a rule, are not discussed. The influence of the nature and concentration of inserted strontium and magnesium on the valence state of a transition element and interatomic interactions remains an enigma.

\footnotetext{
* Corresponding author.

E-mail address: chemdim@mail.ru (D.A. Korolev).
}

Lanthanum gallate doped with bivalent elements and cobalt is much investigated owing to a sufficiently high ionic conductivity $[9,10,14,16-18]$. In this case the data on conductivity appear to differ significantly, strongly depend on the content of diamagnetic additions and cobalt, and are badly reproduced. The authors of $[16,17]$ specify with good reason that only a thoroughly selected concentration of a transition element can provide for a high conductivity and the transport number of oxygen ions close to a unit. We can state with assurance that the same is valid for diamagnetic additions.

It is obvious that the fundamental role in the formation and migration of vacancies in the doped lanthanum gallate belongs to its electronic structure - the state of transition element atoms and interatomic interactions. Systematic study of magnetic properties of the systems containing strontium, magnesium, and $d$-elements as dopants [19-23] showed that a simultaneous doping with a paramagnetic and a diamagnetic element results in an essential clustering in the structure, clusters incorporating transition element atoms, strontium and magnesium, and also the vacancies in the oxygen sublattice. In the case of doping with cobalt and strontium two problems aroused requiring to be solved.

The first problem is associated with the stoichiometry of the solid solution being obtained. The ceramic procedure of the synthesis of complex oxide materials is conventional on obtaining any kinds of construction and functional ceramics. However it has a number of substantial drawbacks associated with the duration of thermal treatment resulting in the batch depletion in volatile 
components and in the composition of the final product differing from the composition of the batch. In particular, in [24] the concentration of cobalt in the system was found to decrease substantially during high-temperature sintering compared to the equation of the solid state reaction. We emphasize that in all the works devoted to the study of cobalt containing systems $[9,10,14,16-18]$ the analysis of the cobalt content after sintering was not carried out, which, to some extent cast some doubt on the quantitative data on optimizing the composition of electron-ionic conductors. Gallium oxide is known to be characterized by a sufficiently high volatility under vacuum [25], which can result in its selective evaporation from the batch and in substantial discrepancies between the composition specified for the synthesis and the obtained composition, and also in the emergence of additional phases.

The second problem is associated with the possibility of various spin states for trivalent cobalt, which must influence interatomic interactions and, consequently, the conductivity of the ceramics.

This work is devoted to a complex study of lanthanum gallate doped with cobalt and magnesium with the ratio $\mathrm{Co}: \mathrm{Mg}=5: 1$ and $2: 1 \quad\left(\mathrm{LaCo}_{x} \mathrm{Mg}_{0.2 x} \mathrm{Ga}_{1-1.2 x} \mathrm{O}_{3-\delta}\right.$ and $\mathrm{LaCo}_{x} \mathrm{Mg}_{0.5 x} \mathrm{Ga}_{1-1.5 x} \mathrm{O}_{3-\delta}$, $x=0.01 \div 0.10$ ). On the basis of mass spectroscopy data a synthetic procedure was developed minimizing the losses of cobalt and gallium oxides during high-temperature sintering. The main aim of the work was to study the states of cobalt atoms and interatomic interactions in the structure and also to reveal their dependence on the relationship between cobalt and magnesium in the systems under study.

\section{Experimental}

Solid solutions of composition $\mathrm{LaCo}_{x} \mathrm{Mg}_{0.2 x} \mathrm{Ga}_{1-1.2 x} \mathrm{O}_{3-\delta}$ and $\mathrm{LaCo}_{x} \mathrm{Mg}_{0.5 x} \mathrm{Ga}_{1-1.5 x}$ $\mathrm{O}_{3-\delta}(x=0.01 \div 0.10)$ were synthesized by ceramic procedure. As starting substances we used special pure grade $\mathrm{La}_{2} \mathrm{O}_{3}$ (99,999\%), $\mathrm{MgO}(99,995 \%), \mathrm{Ga}_{2} \mathrm{O}_{3}$ $(99,999 \%)$, and analytical pure grade $\mathrm{CoO}$ (9999\%). During the high-temperature sintering $(1723 \mathrm{~K})$ of the samples containing strontium and cobalt, as was found in [24], an essential decrease in the concentration of cobalt was observed. This can result from a rapid, during about $2 \mathrm{~h}$ increase in temperature to $1723 \mathrm{~K}$. A selective evaporation of cobalt oxide seems to occur during the high-temperature synthesis.

With the aim to exclude or at least substantially decrease the loss of cobalt it is necessary to select the temperature and time conditions of the synthesis on the basis of the X-ray and quantitative analysis of the end product. The control over the ratio of the components in the solid solution may be carried out with the help of quantitative chemical analysis of both the batch and the obtained end product. This method takes a lot of time, though it is quite efficient. The method of mass spectroscopy control over the losses in the mass of a sample owing to a selective evaporation of the solid solution components that we advance makes possible the determination with a high accuracy: (1) the temperatures of the beginning of the transfer to vapor of all the components of the system under study; (2) the quantity of a component of the system transferred into vapor during a certain period of time.

Therefore, the high temperature mass spectroscopy appears to be the only direct method of analysis making possible the determination of the qualitative and quantitative composition of the vapor with a high accuracy.

The mass spectroscopy experiments were carried out using Knudsen effusion technique combined with mass spectrometric analysis of vapor composition, described in details elsewhere [26], on the mass spectrometer MS 1301 (Construction Bureau, Academy of Science, St. Petersburg). Testing the instrument was conducted by measuring the vapor pressure of calcium fluoride and subsequent comparing the values obtained with reference data [27].

$\mathrm{X}$-ray diffraction experiment for the polycrystalline samples of investigated system have been performed in air at a temperature of $25^{\circ} \mathrm{C}$ using a Bruker "D2 Phaser" diffractometer ( $\mathrm{Cu} \mathrm{K} \alpha$ radiation, Ni filter). The powder data were collected in the $2 \theta$ range of $\left(10-130^{\circ}\right)$ with a step size $0.01^{\circ} 2 \theta$ and counting time $1 \mathrm{~s}$ per step, sample rotation rate of $30 \mathrm{rpm}$. The phase identification was carried out using the Powder Diffraction File database (PDF-2, 2011). Quantitative phase analysis has been performed by the Rietveld method using the TOPAS software complex and the structural data for each of the phases according to the Inorganic Crystal Structure Database (ICSD 2012). The chemical analysis of the content of cobalt and magnesium was carried out by the method of atomic emission spectroscopy on a SPECTRO CIROS, type ISP spectrometer. The error of the analysis did not exceed $4 \%$ from $x$ in the solid solution formula. The preassigned Co:Mg ratio was shown to be preserved by and large as the result of the synthesis. The magnetic susceptibility was measured by Faraday method in the temperature range $77-400 \mathrm{~K}$ at 10 fixed values of magnetic field strength. The accuracy of measuring $\chi_{g}$ is $1 \%$.

\section{Discussion}

\subsection{X-ray study}

The powders of sintered samples at $1723 \mathrm{~K}$, were identified as single-phase with structure of orthorhombic $\mathrm{LaGaO}_{3}$ (S.G. Pbnm) and rhombohedral $\mathrm{LaGaO}_{3}$ (S.G. $R$-3c), which resulted from Rietveld refinement (the profile of the X-ray pattern are given in Fig. 1). As can be seen in Fig. 1 there is a line in the region of $2 \theta \sim 29^{\circ}$, which is a $K_{\beta}$ line. It appears due to the special features of the experiment upon using Ni filter; a shoulder in the most intensive line at $2 \theta \sim 32^{\circ}$ is an edge of the absorption line of Ni filter. $K_{\beta}$ lines were taken into account automatically on treating the patterns in TOPAS software. It

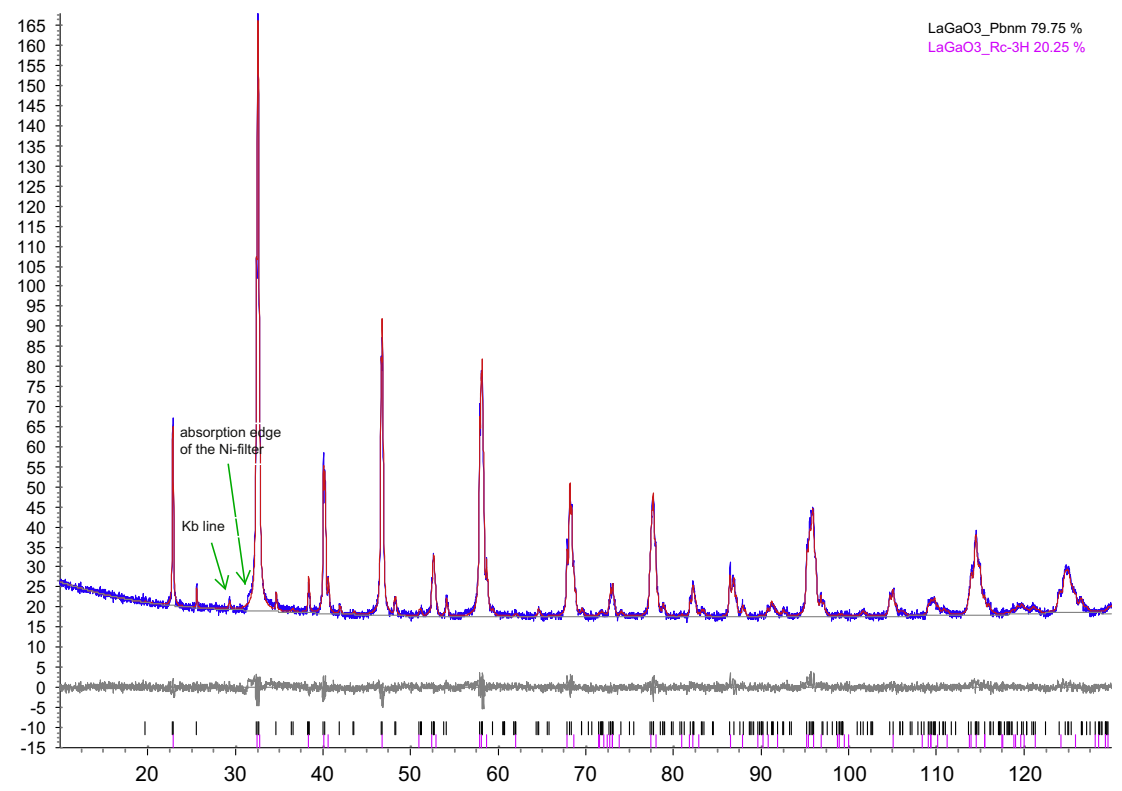

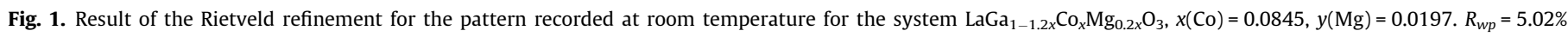
$R_{p}=3.95 \%, \mathrm{GOF}=1.23 \%$, R-Bragg $=1.74$. 

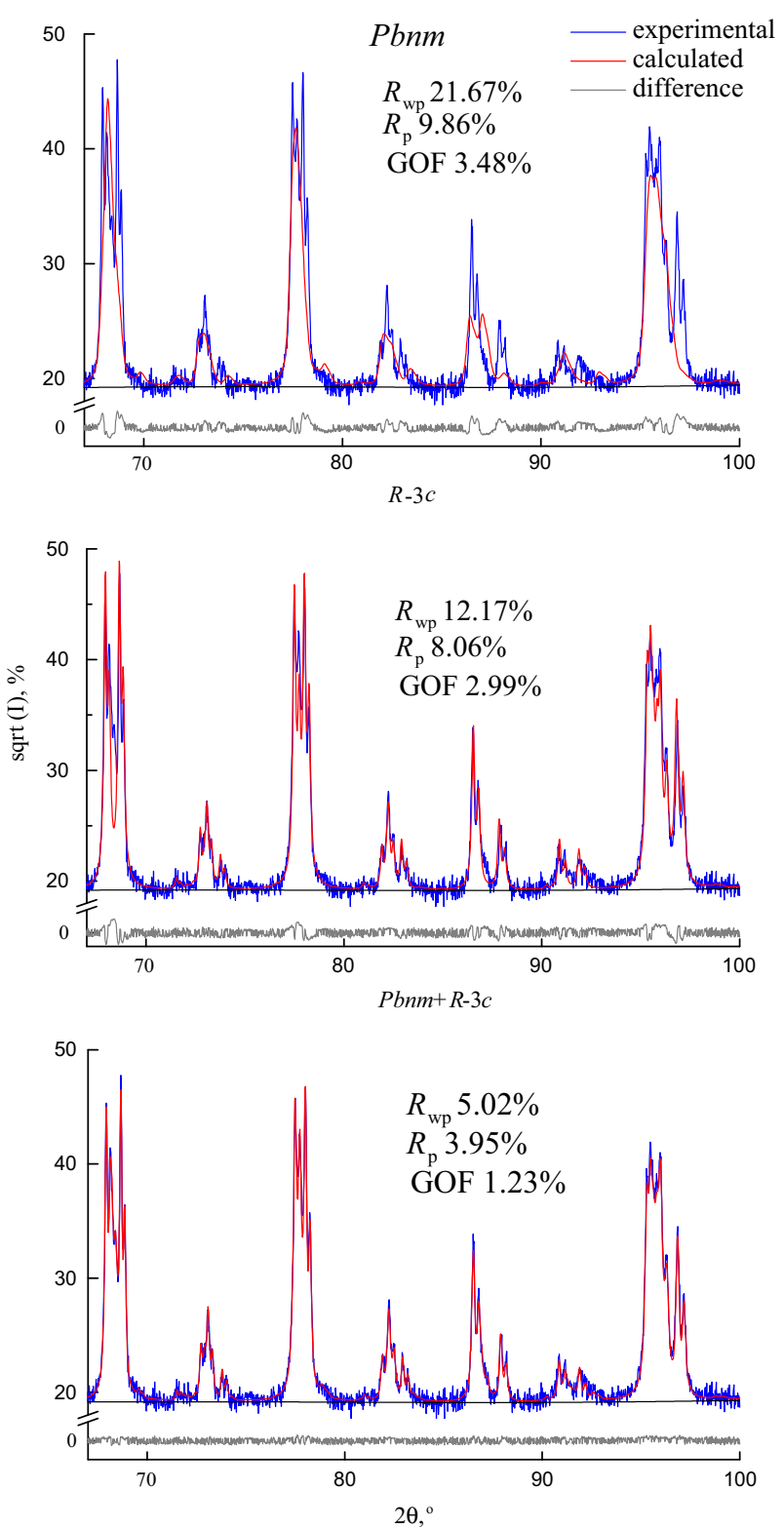

Fig. 2. Calculation of pattern $\mathrm{LaGa}_{1-1.2 x} \mathrm{Co}_{x} \mathrm{Mg}_{0.2 x} \mathrm{O}_{3}, x(\mathrm{Co})=0.0845, y(\mathrm{Mg})=0.0197$ by Rietveld method, using only Pbnm, only $R-3 c$, and upon their joint presence (shows a range of angles $2 \theta=67-100^{\circ}$ ).

is interesting that we were the first to observe the coexistence of two phases of $\mathrm{LaGaO}_{3}$ with different symmetry. In Fig. 2 the calculation of the pattern from Fig. 1 by Rietveld method is shown, which was carried out using Pbnm, $R$-3c, and upon their joint presence. It is seen that the best results are obtained for the last case.

For both systems $\mathrm{Co}: \mathrm{Mg}=5: 1$ and $\mathrm{Co}: \mathrm{Mg}=2: 1$ the solid solutions with low concentrations of doping elements have orthorhombic structure. The mass fraction of rhombohedral phase increases nonlinearly as the quantity $x(\mathrm{Co})+y(\mathrm{Mg})$ increases (Fig. 3). It is seen also that for the system with lower quantity of magnesium $\mathrm{LaCo}_{x} \mathrm{Mg}_{0.2 x} \mathrm{Ga}_{1-1.2 x} \mathrm{O}_{3-\delta}(\mathrm{Co}: \mathrm{Mg}=5: 1)$ the content of rhombohedral phase attains $\sim 60 \%$ at $x(\mathrm{Co})+y(\mathrm{Mg}) \sim 0.11$, whereas for the $\mathrm{LaCo}_{x} \mathrm{Mg}_{0.5 x} \mathrm{Ga}_{1-1.5 x} \mathrm{O}_{3-\delta}(\mathrm{Co}: \mathrm{Mg}=2: 1)$ the quantity of rhombohedral phase at this concentration remains at the level of $\sim 15 \%$. The introduction of greater quantity of magnesium seems to stabilize the low symmetry orthorhombic phase owing to distortions of the structure. This is associated with the difference in the sizes of $\mathrm{Mg}^{2+}$ and $\mathrm{Ga}^{3+}$ atoms $\left(r\left(\mathrm{Mg}^{2+}\right)_{\mathrm{VI}}=0.74 \AA\right.$ vs $\left.r\left(\mathrm{Ga}^{3+}\right)_{\mathrm{VI}}=0.62 \AA\right)$ [28] and the emergence of greater number of oxygen vacancies.

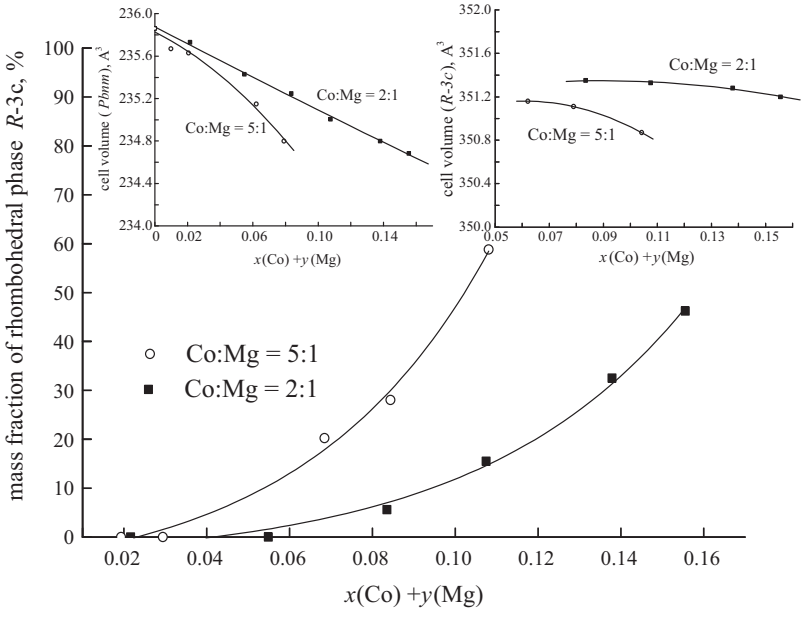

Fig. 3. Mass fraction of rhombohedral phase $(R-3 c) v s$ the doping element content for both systems. In inset: changes in the unit cell volume of orthorhombic and the rhombohedral phases.

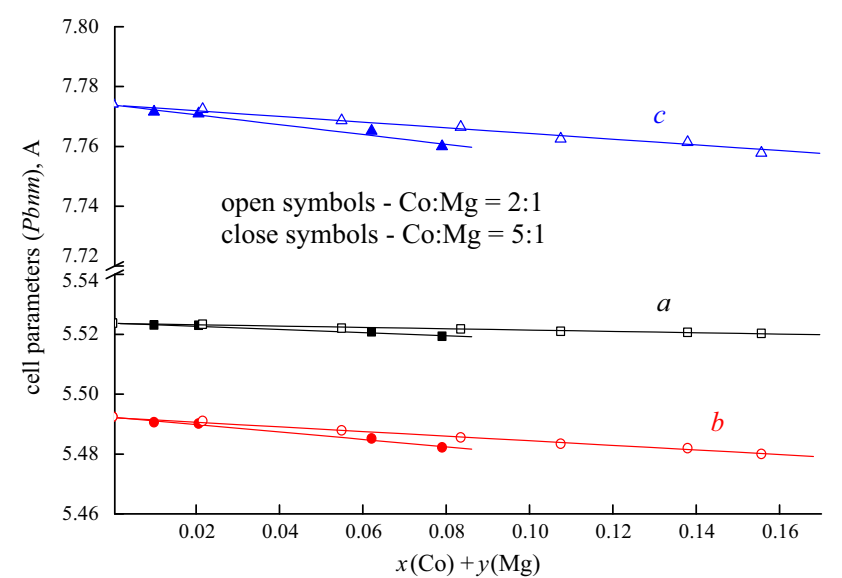

Fig. 4. Plots of the unit cell parameters of orthorhombic phase vs concentration for both systems.

The changes in the unit cell volume for both phases are given in Fig. 3 (in inset). For the $\mathrm{Co}: \mathrm{Mg}=5: 1$ system the unit cell volume of orthorhombic and rhombohedral phases decreases nonlinearly as the quantity of doping elements increases. For the $\mathrm{Co}: \mathrm{Mg}=2: 1$ system we observe almost linear decrease in the unit cell volume of orthorhombic phase, and nonlinear - for rhombohedral phase.

The changes in the unit cell parameters for the Co:Mg = 5:1 and $\mathrm{Co}: \mathrm{Mg}=2: 1$ systems for orthorhombic phase are given in Fig. 4, and for rhombohedral phase - in Fig. 5. The trends in the changes of parameters are similar - in the orthorhombic phase $a, b, c$ monotonously and linearly decrease as the dopant concentration increases, and for rhombohedral phase $a$ parameter decreases nonlinearly and $c$ parameter increases nonlinearly. These data are in good agreement with the data of [29], where the $\mathrm{LaGa}_{1-x-y} \mathrm{Co}_{x} \mathrm{Mg}_{y}$ $\mathrm{O}_{3-\delta}(x=0.6, y=0.1 ; x=0.4, y=0.2)$ solid solutions were studied. The obtained values $(a=5.469 \AA, \quad c=13.175 \AA$; $\quad a=5.492 \AA$, $c=13.249 \AA$ ) are lower than in our work since the level of doping in [29] is essentially higher.

\subsection{Mass spectroscopy investigation}

Partial vapor pressures of the gaseous species were calculated by the ion current comparison method, Eq. (1), using Au as internal pressure standard [30]. 


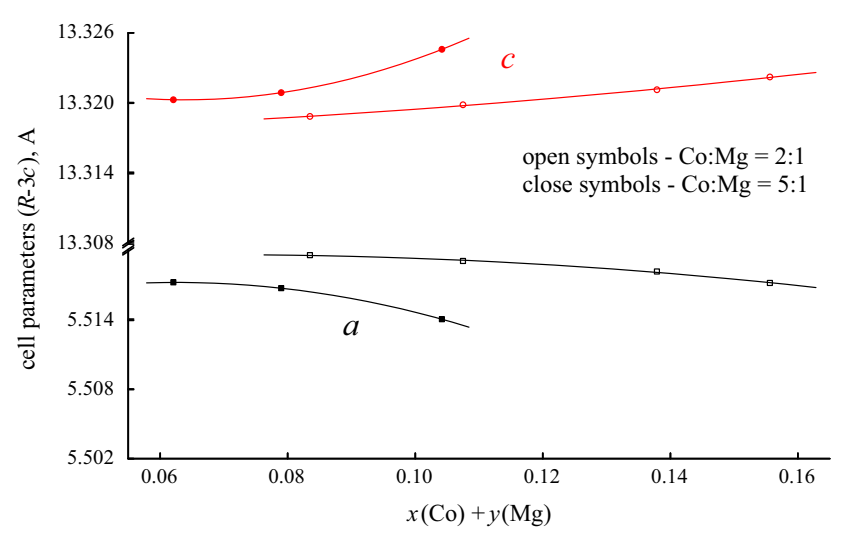

Fig. 5. Plots of the unit cell parameters of rhombohedral phase vs concentration for both systems.

$p_{1}=\frac{p_{2} T_{1} I_{1} \sigma_{2} \gamma_{2}}{T_{2} I_{2} \sigma_{1} \gamma_{1}}$

here $I_{i}$ is the total ion current, $T_{i}$ is the temperature, $\sigma_{i}$ is the ionization cross section, $\gamma_{i}$ is the secondary electron multiplier gain factor, which is known to be proportional to the square root of the molecular mass of the corresponding molecule, $p_{i}$ is a partial pressure of the $i$ species, while " 1 " and " 2 " indexes mean the sample and the standard correspondingly. Molecular cross sections were obtained by the additivity method, atomic cross sections being taken from [31].

The system of heating the effusion cell with the samples allows the operation temperature $(1700 \mathrm{~K})$ to be attained during 2-3 min. Upon rapid heating the mixture of $\mathrm{Ga}_{2} \mathrm{O}_{3}, \mathrm{MgO}, \mathrm{CoO}$ and $\mathrm{La}_{2} \mathrm{O}_{3}$ oxides taken in the necessary stoichiometric ratios the $\mathrm{Ga}_{2} \mathrm{O}^{+}$ion appeared in the mass spectra above the mixture starting from the temperature of about $1450 \mathrm{~K}$. As the temperature increased to the operating $1700 \mathrm{~K}$ a substantial increase in the intensity of the $\mathrm{Ga}_{2} \mathrm{O}^{+}$peak was observed, and the following isothermal exposure resulted in a rapid decrease in the intensity of the ion current of $\mathrm{Ga}_{2} \mathrm{O}^{+}$. This testifies for a sufficiently high rate of depleting the composition of the condensed phase with gallium oxide. Since under real conditions of the synthesis the temperature increases from room to the operating temperature during $1.5-2 \mathrm{~h}$ we decided to carry out the experiment in two stages. In the first stage the effusion cell with the samples was heated to $1400 \mathrm{~K}$ and exposed for $2 \mathrm{~h}$. In the second stage we increased the temperature to $1700 \mathrm{~K}$ and studied the evaporation of the batch and sample.

In the mass spectra above the batch containing $15 \mathrm{~mol} \%$ of cobalt oxide and preheated at $1400 \mathrm{~K}$ the peaks of $\mathrm{Ga}^{+}, \mathrm{Ga}_{2} \mathrm{O}^{+}$ and $\mathrm{Co}^{+}$were detected in the temperature range $1674-1697 \mathrm{~K}$. In order to determine vapor composition, appearance energies of the ions were measured using vanishing current method with $\mathrm{Au}$ as energy standard, ionization energy of which is known to be $9.2 \mathrm{eV}$ [32]. The appearance energies obtained were $( \pm 0.3 \mathrm{eV})$ : $6.0 \pm 0.4\left(\mathrm{Ga}^{+}\right), 7.6 \pm 0.2\left(\mathrm{Ga}_{2} \mathrm{O}^{+}\right)$, and $7.7 \pm 0.4\left(\mathrm{Co}^{+}\right)$. The measurement of appearance energies of these ions showed that they are the products of direct ionization of corresponding molecules, since the measured values agreed within the errors with the ionization energies of atomic gallium, of $\mathrm{Ga}_{2} \mathrm{O}$ molecule, and of atomic cobalt respectively [32]. Therefore we can state that on heating the oxide mixture containing $15 \mathrm{~mol} \%$ of cobalt oxide at the temperature of about $1700 \mathrm{~K}$ atomic gallium and cobalt, and also $\mathrm{Ga}_{2} \mathrm{O}$ and oxygen are evaporated according to Eqs. (2)-(4).

$\mathrm{Ga}_{2} \mathrm{O}_{3}($ sol $)=\mathrm{Ga}_{2} \mathrm{O}$ (gas) $+\mathrm{O}_{2}$ (gas)

$\mathrm{Ga}_{2} \mathrm{O}_{3}($ sol $)=2 \mathrm{Ga}($ gas $)+3 / 2 \mathrm{O}_{2}($ gas $)$
$\mathrm{CoO}(\mathrm{sol})=\mathrm{Co}($ gas $)+1 / 2 \mathrm{O}_{2}($ gas $)$

The partial pressure of oxygen, according to Eqs. (2)-(4), may be calculated from one of Eqs. (5)-(7), which take into account the fact that there is an aperture in the effusion cell, and the system is in the state of dynamic equilibrium a condensed phase - vapor.

$$
\begin{aligned}
p\left(\mathrm{O}_{2}\right) & =p\left(\mathrm{Ga}_{2} \mathrm{O}\right)\left[M\left(\mathrm{O}_{2}\right) / M\left(\mathrm{Ga}_{2} \mathrm{O}\right)\right]^{0.5}+p\left(\mathrm{O}_{2}\right) \\
& =0.75 p(\mathrm{Ga})\left[\mathrm{M}\left(\mathrm{O}_{2}\right) / \mathrm{M}(\mathrm{Ga})\right]^{0.5}+p\left(\mathrm{O}_{2}\right) \\
& =0.5 p(\mathrm{Co})\left[M\left(\mathrm{O}_{2}\right) / M(\mathrm{Co})\right]^{0.5}
\end{aligned}
$$

Here $M$ is the molar mass.

We stopped the experiment after the partial pressures of Ga, $\mathrm{Ga}_{2} \mathrm{O}$, and atomic cobalt decreased substantially, which resulted in the changes in the mole ratios in the sample. We emphasize that upon isothermic exposure at the temperature of about $1700 \mathrm{~K}$ the ratio of the intensities of ion currents $\mathrm{Ga}^{+} / \mathrm{Ga}_{2} \mathrm{O}^{+}$gradually increased, which testifies for an increase in the partial pressure of atomic gallium relative to the partial pressure of $\mathrm{Ga}_{2} \mathrm{O}$.

When measuring partial pressures of the vapor species the method of complete isothermal vaporization can also be used. The pressure inside the effusion cell is associated with the mass loss and is expressed by Hertz-Knudsen transformed Eq. (6):

$p_{i}=\frac{q_{i}}{s t L} \sqrt{\frac{2 \pi R T}{M_{i}}}$

where $p$ is the partial pressure; $q$ the mass of a substance evaporated through the effusion aperture of area $s$ with Clausing factor $L$ during the time $t ; R$ is the universal gas constant; $T$ the temperature, K; $M_{i}$ the molecular mass of an I component in the vapor.

Hertz-Knudsen equation may be rewritten as Eq. (7):

$\frac{m}{s t}=\frac{p L}{2285 \sqrt{T / M_{i}}}$

The value 2285 is associated with the fact that partial pressures are measured in Pascal. The left part of Eq. (5) is nothing but the rate of $i$-component evaporation. In our case, given a known area of the effusion aperture equal to the area of evaporation, we can calculate the rate of atomic cobalt evaporation recalculating the value obtained from Eq. (9) per the evaporation area equal to $1 \mathrm{~cm}^{2}$ and the evaporation time $-1 \mathrm{~h}$.

Since the synthesis of a sample from the batch is carried out at atmospheric pressure, not in vacuum, the obtained values may be recalculated with respect to the partial pressure of oxygen in air being 21,275 Pa. For reactions (2)-(4) we wrote the expressions for the equilibrium constants as Eqs. (8)-(10),

$K_{p}(2)=\frac{p\left(\mathrm{Ga}_{2} \mathrm{O}\right) p\left(\mathrm{O}_{2}\right)}{a\left(\mathrm{Ga}_{2} \mathrm{O}_{3}\right)}$

$K_{p}(3)=\frac{p^{2}\left(\mathrm{Ga}_{2} \mathrm{O}\right) p^{1.5}\left(\mathrm{O}_{2}\right)}{a\left(\mathrm{Ga}_{2} \mathrm{O}_{3}\right)}$

$K_{p}(4)=\frac{p(\mathrm{Co}) p^{0.5}\left(\mathrm{O}_{2}\right)}{a(\mathrm{CoO})}$

where $a\left(\mathrm{Ga}_{2} \mathrm{O}_{3}\right), a(\mathrm{CoO})$ are the activities of gallium and cobalt oxides in the condensed phase respectively.

The plots of evaporation rates of $\mathrm{Ga}_{2} \mathrm{O}_{3}$ and $\mathrm{CoO}$ in air vs the time of evaporation at the temperature $1700 \mathrm{~K}$ is given in Fig. 6 . The starting composition of the batch is $\mathrm{La}_{2} \mathrm{O}_{3}-\mathrm{Ga}_{2} \mathrm{O}_{3}-x \mathrm{CoO}-$ $y \mathrm{MgO}, x=0.15, y=0.5 \mathrm{x}$. On synthesis in the air atmosphere the rate of cobalt oxide evaporation (given $15 \mathrm{~mol} \%$ of $\mathrm{CoO}$ in the batch) more than twice exceeds the rate of $\mathrm{Ga}_{2} \mathrm{O}_{3}$ evaporation. The plots of the evaporation rates of $\mathrm{Ga}_{2} \mathrm{O}_{3}$ in air vs the evaporation time at the temperature $1700 \mathrm{~K}$ are given in Fig. 6 (in inset). The starting 


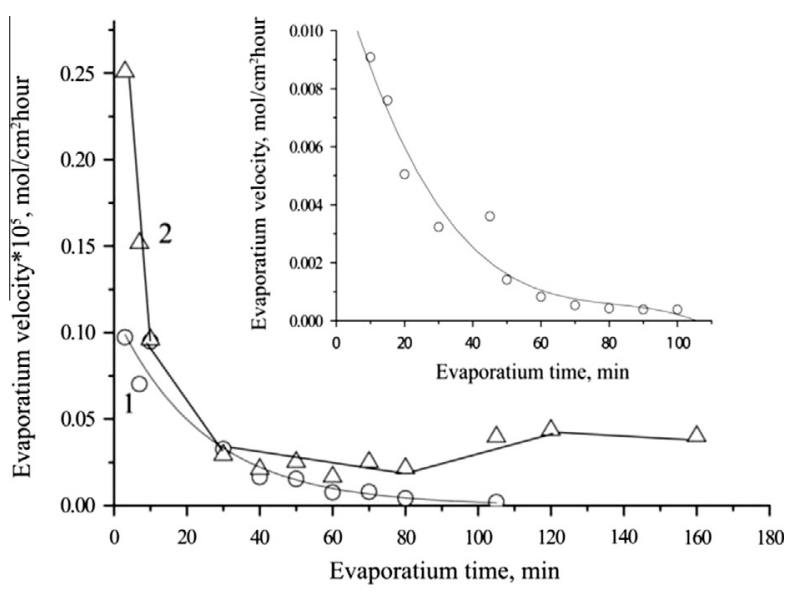

Fig. 6. The plots of evaporation rates of $\mathrm{Ga}_{2} \mathrm{O}_{3}$ and $\mathrm{CoO}$ in air vs the time: $1-\mathrm{Ga}_{2} \mathrm{O}_{3}$ 2 - CoO. In inset: the plots of the evaporation rates of $\mathrm{Ga}_{2} \mathrm{O}_{3}$ in air $v s$ the time at the temperature $1700 \mathrm{~K}$.

composition of the batch is $\mathrm{La}_{2} \mathrm{O}_{3}-\mathrm{Ga}_{2} \mathrm{O}_{3}-x \mathrm{CoO}-y \mathrm{MgO}, x=0.01$, $y=0.5 x$.

Cobalt oxide is less volatile, which at the temperature of the synthesis of doped lanthanum gallate can transfer to vapor as atomic cobalt and molecular oxygen. Oxygen of the air shifts the "condensed phase - vapor" equilibrium in such a manner that on heating in air it is cobalt oxide that appears a more volatile component.

Using the results of mass spectroscopy analysis we carried out a step by step synthesis of the solid solutions. Thoroughly ground and pressed into pellets oxide mixture was fed into corundum crucibles and put into the furnace. Its temperature was gradually increased to $1073 \mathrm{~K}$. Then we successively increased the temperature by $373 \mathrm{~K}$ each $5 \mathrm{~h}$. As the temperature attained $1723 \mathrm{~K}$, all the samples were sintered for $52 \mathrm{~h}$ to reach the equilibrium. In such a manner we were able to minimize the losses of cobalt. The chemical analysis showed that in the obtained solid solutions the content of cobalt is closer to the predetermined content $(x$ by the batch 0.10 ; according to the analysis 0.845 for the $\mathrm{Co:Mg}=5: 1$, and 0.895 for $\mathrm{Co}: \mathrm{Mg}=2: 1$ system).

\subsection{Magnetic measurements}

The magnetic susceptibility of the solid solutions was found to depend on the strength of magnetic field for both systems over the whole concentration range. This dependence for magnetically diluted systems seems to point to the existence of highly nuclear clusters of paramagnetic atoms and must not be associated with a long range order in the substance. An examination of the dependencies of magnetization suggests a super paramagnetic behavior of our systems. As can be seen from Fig. 7, where the dependence of molar magnetization on $H / T$ is given, the magnetization at various temperatures and fields appears to be the same. The observed dependence is typical for all the solutions under study. This is one of two requirements for superparamagnetic behavior [33-36]. The second requirement is the absence of hysteresis loop.

According to the theory of paraprocess in the ferromagnets $[37,38]$ the plot in the coordinates $H / M-M^{2}$ must be linear and obey Eq. (11):

$H=\alpha M+\beta M^{3}$

In this case the deviations from the linear character are associated with the special features of the spin system behavior in the magnetic field. The construction of Belov-Arrott curves allows a definite conclusion to be made that the spontaneous magnetization is

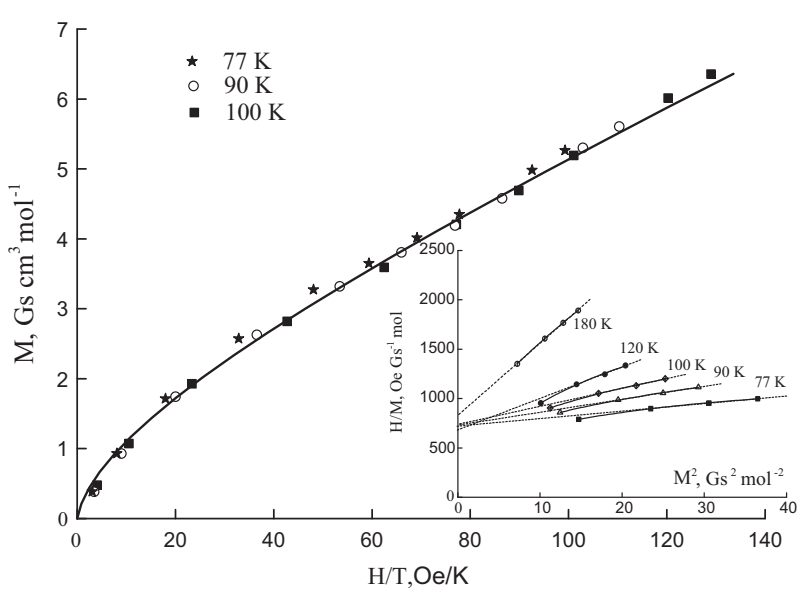

Fig. 7. Plot of magnetization vs $\mathrm{H} / \mathrm{T}$ for $\mathrm{LaCo}_{x} \mathrm{Mg}_{0.5 x} \mathrm{Ga}_{1-1.5 x} \mathrm{O}_{3}$ system $(x(\mathrm{Co})=0.0359)$. In inset: Belov-Arrott curves for same sample.

absent in our systems, since the thermodynamic coefficient $\alpha$ is positive for all the temperatures, which is also typical for all the systems under study (Fig. 7 (in inset)).

Therefore we may suggest the existence of highly nuclear clusters of cobalt atoms in the systems under study.

To calculate the paramagnetic component of magnetic susceptibility we used $\chi_{g}$ extrapolated to the infinitely large field strength $(1 / H=0)$. The matter is that we measure all the susceptibilities at eleven values of applied magnetic field (from 9000 Oe to 300 Oe), and received linear plots $\chi_{g}=f(1 / H)$. The plots of inverse paramagnetic components of magnetic susceptibility vs temperature are given in Fig. $8 \mathrm{a}$ and $\mathrm{b}$.

These dependencies demonstrate a deviation from Curie-Weiss law at low and high temperatures, the deviation in the region of high-temperatures increasing as cobalt concentration increases. Such deviations of magnetic susceptibility from Curie-Weiss law at high-temperatures is typical for Pauli temperature independent paramagnetism, i.e. the paramagnetism of free electrons. Pauli paramagnetism can arise from the following: as was shown in [39], $e_{g}$ electron levels in lanthanum gallate doped with $3 d$-elements are located within the gap between the valence and conduction band of $\mathrm{LaGaO}_{3}$. However, the deviations of the dependence of inverse magnetic susceptibility from the Curie-Weiss law at high-temperatures may arise also from the spin transitions low spin $\leftrightarrow$ intermediate spin $\leftrightarrow$ high spin states of $\mathrm{Co}$ (III). Therefore it would be improper to estimate the values of Pauli paramagnetism since the obtained values would bear no physical significance.

The deviations of the plot $1 / \chi_{\text {co }}-T$ from Curie-Weiss law at low temperatures are most probably determined by the character of exchange interactions between cobalt atoms and as a rule are typical for ferrimagnetics. This is the first evidence for a competition between ferromagnetic and antiferromagnetic exchange interactions in the systems under study with those first being dominant.

Concentration dependences of paramagnetic component of magnetic susceptibility and temperature dependences of the effective magnetic moment for both systems are given in Figs. 9 and 10 respectively. To show the run of the isotherms we plotted the curves calculated as polynomials fitting them to the experimental points by the least square method.

It follows from the shape of isotherms of magnetic susceptibility that the exchange interactions are antiferromagnetic in the solid solutions under study - the magnetic susceptibility decreases as the content of cobalt increases. It must be emphasized in this case that the isotherms are located substantially higher than the isotherms for $\mathrm{LaCo}_{x} \mathrm{Ga}_{1-x} \mathrm{O}_{3}$ containing no magnesium and they lie the higher, the greater is the content of magnesium in the system. 

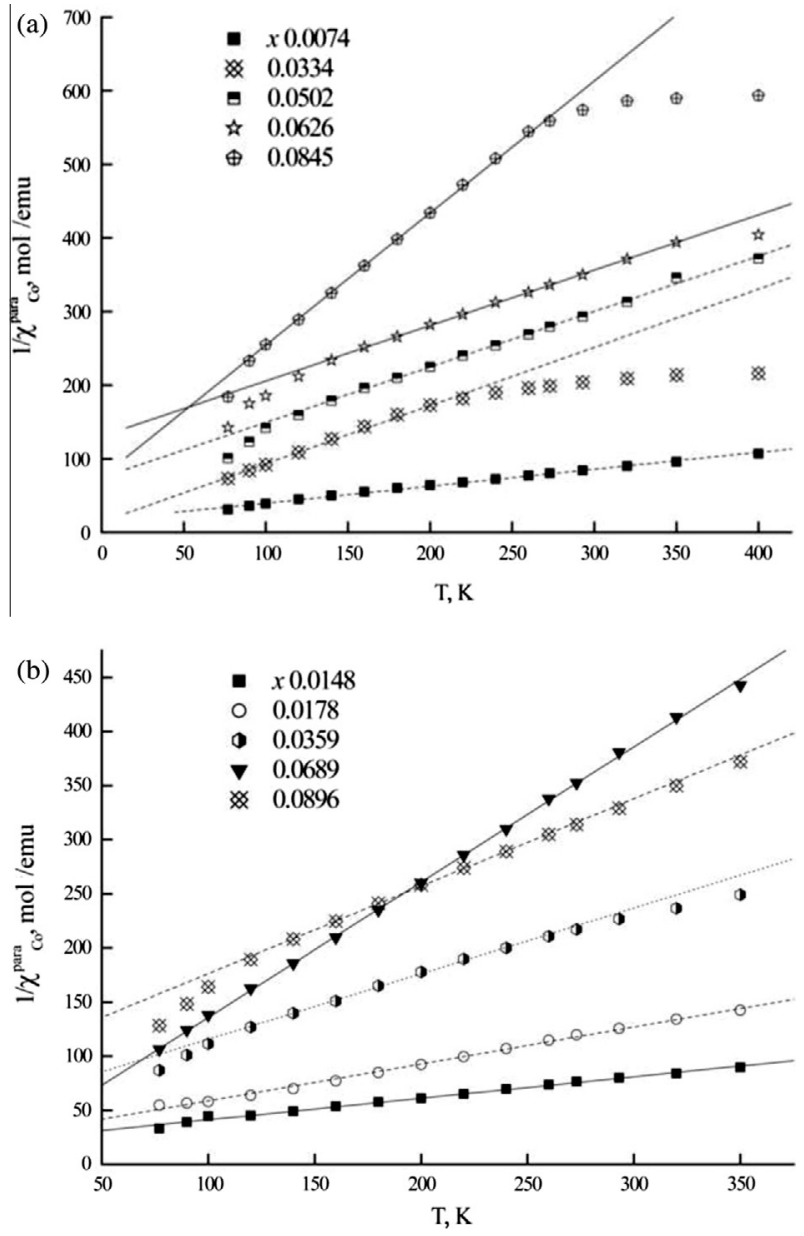

Fig. 8. (a) and (b) Temperature dependencies of $1 / \chi^{\text {para }}$ for: (a) $\mathrm{LaGa}_{1-1.2 x} \mathrm{Co}_{x} \mathrm{Mg}_{0.2^{-}}$ ${ }_{x} \mathrm{O}_{3}$ system; (b) $\mathrm{LaCo}_{x} \mathrm{Mg}_{0.5 x} \mathrm{Ga}_{1-1.5 x} \mathrm{O}_{3}$ system.

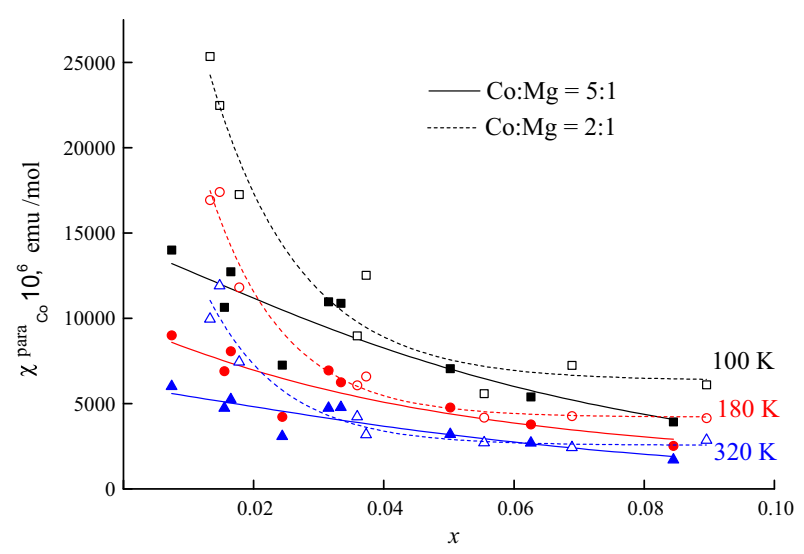

Fig. 9. Plot of $\chi^{\text {para }}$ vs cobalt concentration ( $x$ ) for various Co-containing systems.

Of greatest interest for judging about the interatomic interactions in the solid solutions under study are the temperature dependences of the effective magnetic moment extrapolated to the infinite dilution and their comparison with cobalt containing systems studied previously - $\mathrm{LaCo}_{x} \mathrm{Ga}_{1-x} \mathrm{O}_{3}$ and $\mathrm{La}_{1-0.2 x} \mathrm{Sr}_{0,2 x} \mathrm{Co}_{x} \mathrm{Ga}_{1-x} \mathrm{O}_{3}$ [24]. Fig. 10 gives also theoretical dependences of the effective magnetic moment for single cobalt atoms in the degree of oxidation +2 and +3 , and also for $\mathrm{Co}(\mathrm{III})$ in the state with an intermediate spin $S=1,3 T 1 \mathrm{~g}$. The matter is that $\mathrm{Co}(\mathrm{III})$ in an octahedron of oxygen atoms can be in three ground states: $S=0,{ }^{1} A_{1 g}$ - low spin;

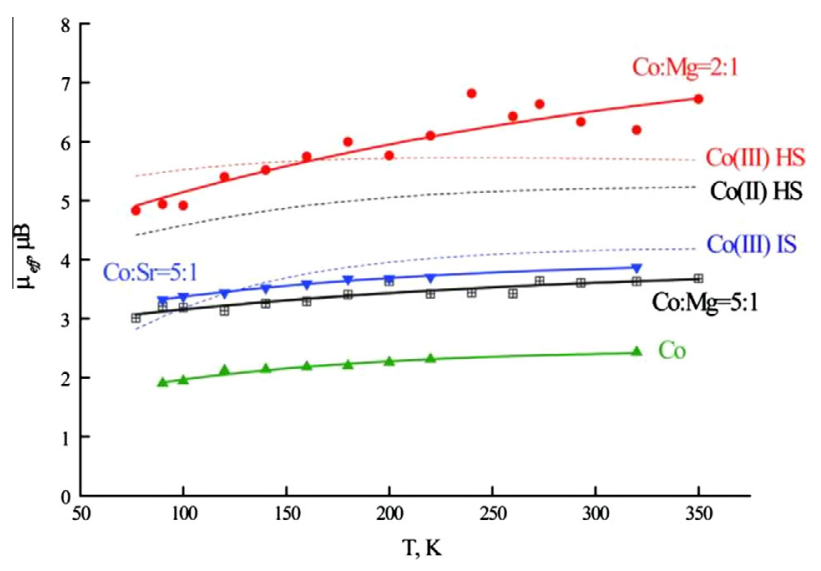

Fig. 10. Plot of $\mu_{\text {eff }} v s$ temperature at the infinite dilution for various Co-containing systems.

$S=2,{ }^{5} T_{2 g}-$ high spin, and substantial distortions of the nearest surrounding, which may be expected in our systems owing to the oxygen vacancies and greater magnesium atoms in the same sites as cobalt, may result in a state with intermediate spin $S=1$, ${ }^{3} T_{1 \mathrm{~g}}[40,41]$.

It is seen from Fig. 10 that for the ratio Co:Mg $=2: 1$ the effective magnetic moment cannot be ascribed to single cobalt atoms, whatever valence state they were in. The effective magnetic moment exceeds the only spin value especially at high-temperatures. For the ratio Co:Mg $=5: 1 \mu_{\text {eff }}$ is lower and almost coincide with $\mu_{\text {eff }}$ obtained for strontium containing systems $\mathrm{Co}: \mathrm{Sr}=5: 1$ [24] and were interpreted as fitting single cobalt atoms partially in the low spin state and partially in the state of spin equilibrium $\left({ }^{1} A_{1 g} \leftrightarrow{ }^{5} T_{2 g}\right)$. However we cannot describe the systems containing magnesium within the same approach as in [24], since we found that in our solid solutions the magnetic susceptibility depends on the magnetic field. In other words, the dependence of magnetic susceptibility on the magnetic field strength and the values of effective magnetic moment at infinite dilution testify for the fact that even in much diluted solid solutions there remain the high-nuclearity clusters containing also magnesium atoms and vacancies in the oxygen sublattice along with cobalt atoms.

We emphasize that in the studies of lanthanum gallate doped with cobalt only and lanthanum gallate doped with cobalt and strontium - $\mathrm{LaCo}_{x} \mathrm{Ga}_{1-x} \mathrm{O}_{3}$ and $\mathrm{La}_{1-0.2 x} \mathrm{Sr}_{0.2 x} \mathrm{Co}_{x} \mathrm{Ga}_{1-x} \mathrm{O}_{3}$ [24] no dependence of magnetic susceptibility on magnetic field was observed, whereas such dependence appeared also in the systems containing chromium and magnesium [20]. This fact allowed us to suggest that in both kinds of the systems (containing chromium and cobalt) we have large clusters of paramagnetic atoms. A substantial difference in the magnetic characteristics of the systems doped with paramagnetic atom only and doped in addition with bivalent element points to the fact that the atoms of bivalent element are included into these clusters, since their concentration is small compared to the concentration of paramagnetic atoms and otherwise they could not so drastically influence the magnetic characteristics of diluted solid solutions. But the presence of a bivalent element in the lattice results in vacancies in the oxygen sublattice, and these vacancies must be located in the vicinity of bivalent atoms. Therefore, they also must be included into the clusters.

Within the clusters the exchange interactions are predominantly ferromagnetic with a certain contribution of antiferromagnetic exchange, i.e. by and large we deal with ferrimagnetic behavior. The conclusion about the domineering ferromagnetic component in the interaction between cobalt atoms is associated with the fact that the character of temperature dependence of the effective magnetic moment and its high absolute values cannot 
be ascribed to antiferromagnetic type of interactions. Strong ferromagnetic exchange may be associated with the fact that rather strong local distortions of cobalt surrounding appear near a vacancy within a cluster. It is magnesium that seems to provoke the formation of highly nuclear clusters of paramagnetic atoms owing to the special features of its location in the structure of lanthanum gallate - magnesium atoms are in the same crystallographic sites as gallium atoms and paramagnetic atoms. In such a case not only large magnesium atoms but also the vacancies in the oxygen sublattice immediately connected to magnesium atoms result in a stronger local distortion of the structure (compared to strontium containing systems) and, in their turn, to a destabilization of doped lanthanum gallate. Cobalt atoms seem to help in the stabilization of the whole system at the expense of cobalt atom clustering round the vacancies to give highly nuclear clusters with cobalt atoms linked by magnetic superexchange interactions.

In a distorted crystal field the probability of the realization of intermediate spin state increases [40,41]. The exchange interactions between cobalt atoms with different spins must be ferromagnetic [42]. As the concentration of cobalt increases, paramagnetic atoms appear in the vicinity of clusters and interact antiferromagnetically between themselves and with the clusters, which results in a shape of magnetic susceptibility isotherms typical for antiferromagnetics.

We emphasize also that clustering in the solid solutions increases as the fraction of a diamagnetic component with respect to cobalt increases. It is definitely more pronounced for magnesium containing systems than for the systems with strontium, which seems to be associated with magnesium location in the structure of lanthanum gallate and its stronger effect on the orbitals of oxygen atoms.

\section{Conclusions}

By the data of X-ray for the first time different modifications of $\mathrm{LaGaO}_{3}$ were found in the systems under study - orthorhombic and rhombohedral depending on the level of doping. As the content of doping elements increases, the unit cell volume decreases, which can be associated with the emergence of oxygen vacancies and the transfer of cobalt(III) atoms from high spin state to the intermediate and low spin states. The study of evaporation in the $\mathrm{CoO}-\mathrm{La}_{2} \mathrm{O}_{3}-\mathrm{MgO}-\mathrm{Ga}_{2} \mathrm{O}_{3}$ system resulted in the conclusion that under the conditions of ceramic high-temperature synthesis in air gallium oxide almost does not evaporate. Taking into account the fact that gallium oxide comprises the greater part of perovskite structure we may state that the stoichiometry of the obtained doped lanthanum gallate is fairly influenced by the loss of gallium. At the same time the losses of cobalt during the synthesis may appear significant. The chemical analysis of the obtained solid solutions illustrated emphatically that varying the conditions of the synthesis the samples may be obtained differing to a particular extent from the preassigned composition. This allowed us to develop the synthetic procedure diminishing the losses of cobalt. Since it is completely evident that the changes in the composition influence all the physical and chemical characteristics of the systems under study, in the conductivity among them, we must strongly recommend that the researches of cobalt containing systems carry out the chemical analysis of its content after sintering for obtaining reproducible results.

The study of magnetic properties of lanthanum gallate doped with cobalt and magnesium proves once more that heterovalent doping results in strong cluster formation, the clusters including paramagnetic atoms, the atoms of bivalent element, and vacancies in the oxygen sublattice associated with them. Clustering seems to stabilize the defect structure, and the formation of a certain electron structure providing for an unusual rigidity of such clusters favors the realization of paramagnetic ground states of trivalent cobalt even though the presence of magnesium in the neighboring sites increases the strength of the crystal field.

\section{Acknowledgment}

XRD studies were performed at Center for X-ray Diffraction Studies of St. Petersburg State University.

\section{References}

[1] T. Ishihara, H. Matsuda, Y. Takita, J. Am. Chem. Soc. 116 (1994) 3801-3803. D..

[2] T. Ishihara, T. Yamada, H. Arikawa, H. Nishiguchi, Y. Takita, Solid State Ionics 135 (2000) 631-636.

[3] N. Trofimenko, H. Ullmann, Solid State Ionics 118 (1999) 215-227.

[4] P. Datta, P. Majewski, F. Aldinger, J. Alloys Comp. 438 (2007) 232-237.

[5] M. Kajitani, M. Matsuda, A. Hoshikawa, S. Harjo, T. Kamiyama, T. Ishigaki, F. Izumi, M. Miyake, J. Phys. Chem. Sol. 68 (2007) 758-764.

[6] N.J. Long, F. Lecarpentier, H.L. Tuller, J. Electroceram. (1999) 399-407.

[7] J. Xue, Yu. Shen, Q. Zhou, T. He, Y. Han, Int. J. Hydrogen Energy 35 (2010) 294300.

[8] J.Y. Yi, G.M. Choi, Solid State Ionics 148 (2002) 557-565.

[9] R. Polini, A. Falsetti, E. Traversa, O. Schaf, P. Knauth, J. Eur. Ceram. Soc. 27 (2007) 4291-4296.

[10] T. Ishihara, S. Ishikawa, K. Hosoi, H. Nishiguchi, Y. Takita, Solid State Ionics 175 (2004) 319-322.

[11] T. Ishihara, T. Akbay, H. Furutani, Y. Takita, Solid State Ionics 113-115 (1998) $585-591$.

[12] H. Ullmann, N. Trofimenko, Solid State Ionics 119 (1999) 1-8.

[13] R.T. Baker, B. Gharbage, F.M.B. Marques, J. Eur. Ceram. Soc. 18 (1998) 105-112.

[14] N. Trofimenko, H. Ullmann, Solid State Ionics 124 (1999) 263-270.

[15] M.T. Colomer, J.A. Kilner, Solid State Ionics 182 (2010) 76-81.

[16] T. Ishihara, S. Ishikawa, M. Ando, H. Nishiguchi, Y. Takita, Solid State Ionics 173 (2004) 9-12.

[17] M. Yashima, K. Nomura, H. Kageyama, Y. Miyazaki, N. Chitose, K. Adachi, Chem. Phys. Lett. 380 (2003) 391-396.

[18] S. Wang, R. Zheng, A. Suzuki, Solid State Ionics 174 (2004) 157-162.

[19] N.V. Chezhina, D.A. Korolev, S.M. Sukharzhevskii, O.V. Glumov, Russ. J. Gen. Chem. 80 (5) (2010) 915-919.

[20] N.V. Chezhina, D.A. Korolev, Solid State Ionics 225 (2012) 201-205.

[21] D.A. Korolev, N.V. Chezhina, Russ. J. Gen. Chem. 81 (10) (2011) 206-210.

[22] N.V. Chezhina, D.A. Korolev, Russ. J. Gen. Chem. 84 (3) (2012) 353-359.

[23] D.A. Korolev, N.V. Chezhina, Zh.A. Lytkina, Russ. J. Gen. Chem. 84 (3) (2012) 360-365.

[24] N.V. Chezhina, E.V. Zharikova, M.N. Knyazev, Russ. J. Gen. Chem. 80 (12) (2010) 2399-2404.

[25] E.K. Kazenas, Yu.V. Tsvetkov, Thermodynamics of Evaporation of Oxides, first ed., LKI publisher, Moscow, 2008 (in Russian).

[26] G.A. Semenov, E.N. Nikolaev, K.E. Frantseva, Application of Mass Spectrometry in Inorganic Chemistry, Khimiya, Leningrad, 1976 (in Russian).

[27] V.P. Glushko (Ed.), Thermodynamic properties of individual substances, Handbook, third ed., vol. 21, Nauka, Moscow, 1979 (No. 1, in Russian).

[28] R.D. Shannon, Acta Crystallogr. A32 (1976) 751.

[29] A.A. Yaremchenko, V.V. Kharton, A.P. Viskup, E.N. Naumovich, V.N. Tikhonovich, N.M. Lapchuk, Solid State Ionics 120 (1999) 65-74.

[30] R.C. Paule, J. Mandel, Pure Appl. Chem. 31 (1972) 371-394.

[31] J.B. Mann, Recent development in mass spectrometry, in: K. Ogata, T. Hayakawa (Eds.), University Park Press, Baltimore, 1970.

[32] V.N. Kondratiev (Ed.), Dissociation energy of chemical bonds. Potentials of ionization and the electron affinity, Handbook, Nauka, Moscow, 1974 (in Russian).

[33] A. Bajpai, A. Banerjee, Phys. Rev. B 62 (2000) 8996-9004.

[34] C.P. Bean, J.D. Livingston, J. Appl. Phys. 10 (1959) 120 S.

[35] Y. Ishikawa, J. Phys. Soc. Jpn. 17 (1962) 1835-1840.

[36] K.P. Belov, Magnetic Transformations, GIFML, Moscow, 1959 (in Russian).

[37] D.G. Kellerman, E.V. Shalaeva, A.I. Gusev, Phys. Solid State 46 (9) (2004) 16861692.

[38] N.V. Chezhina, E.V. Bodritskaya, N.A. Zhuk, V.V. Bannikov, I.R. Shein, A.L. Ivanovskii, Phys. Solid State 50 (11) (2008) 2121-2126.

[39] R.L. Martin, A.H. White, The nature of the transition between high-spin and low-spin octahedral complexes of the transition metals, Transition Metal Chemistry, Dekker, New York, 1968.

[40] B. Buffat, G. Demazeau, M. Pouchard, P. Hagenmuller, Mater. Res. Bull. 18 (9) (1983) 1153-1158

[41] G. Demazeau, M. Pouchard, M. Tomas, J.-F. Colombet, J. Grenier, L. Fournes, J.-L. Soybeyroux, P. Hagenmuller, Mater. Res. Bull. 15 (5) (1980) 451-459.

[42] V.T. Kalinnikov, Yu.V. Rakitin, Introduction on magnetochemistry, first ed., The Static Magnetic Susceptibility Method, Nauka, Moscow, 1980 (in Russian). 